\title{
Energy deposition and dose enhancement using Monte Carlo derivative sampling: applications in brachytherapy
}

\author{
H Khan', ZU Koreshi ${ }^{2}$, U Aziz ${ }^{3 *}$, SR Sheikh ${ }^{3}$ and SA Ahmad ${ }^{4}$ \\ 'Department of Sciences \& Humanities, National University of Computer and Emerging Sciences, Islamabad, Pakistan. \\ ${ }^{2}$ Faculty of Graduate Studies, Air University, Islamabad, Pakistan. \\ ${ }^{3}$ Department of Mechatronics Engineering, Air University, Islamabad, Pakistan. \\ ${ }^{4}$ Department of Strategic Studies, National Defence University, Islamabad, Pakistan.
}

\begin{abstract}
Energy deposition and radiation dose distribution by the use of gold, a high-Z biocompatible element in water solution, is estimated as a function of source energy typical of brachytherapy sources $(15 \mathrm{keV}, 20 \mathrm{keV}, 30 \mathrm{keV}, 40 \mathrm{keV}, 80$ $\mathrm{keV}, 90 \mathrm{keV}, 150 \mathrm{keV}, 300 \mathrm{keV}, 1 \mathrm{MeV}$ ), solution concentration (5-25 mg Au/g $\mathrm{H}_{2} \mathrm{O}$ ), and solution placement (1-2 cm concentric shells). Monte Carlo (MC) simulations are carried out with the MCNP5 code, compared with other widely used MC codes such as PENELOPE and GEANT, to validate the dose estimates, which may vary considerably due to artifacts and data libraries, and extended to a sensitivity analysis using perturbation estimates. The energy deposition and radiation dose are estimated as a function of monoenergetic source radiation energy, and concentration of gold in solution. MC simulation is carried out in the coupled photon-electron radiation mode for x-rays emanating from a radiation source implanted in a water cell, for which results are valid for a cancer cell modelled by a spherical water phantom. For carrying out sensitivity studies, the Monte Carlo perturbation feature with material perturbations was used to sample derivatives in a single run which were used in a Taylor series to estimate both dose and Dose Enhancement Factor (DEF) from single MC runs. Close agreement was found between dose estimates from MCNP5, PENELOPE and GEANT, in spite of artifacts such as cut-offs in electron transport. It was also found that dose increases with energy of a source, and that dose enhancement, for a given concentration, decreases with source energy. The perturbation estimates result in enhanced computational efficiency.
\end{abstract}

Keywords: Brachytherapy, dose enhancement, energy deposition, gold nanoparticles, Monte Carlo simulation.

\section{INTRODUCTION}

Energy deposition from radiation is of use for several engineering and medical applications, such as the design of radiation shields and in the treatment of cancer. This research carried out simulations to estimate the dose in a water cell similar to a cancer cell in composition. In nuclear medicine, radiation nuclide therapy (RNT) is used for the treatment of organs such as the thyroid, bones, heart, liver and for the treatment of cancer in organs such as the lungs, breast, colon and rectum, prostate, stomach, and liver. Nuclear radiation, in the form of X-rays, gamma rays and charged particles, can damage the DNA (deoxyribonucleic acid) molecules of cancer cells that carry the genetic information and pass it from one generation to the next, to stop their further division. RNT is used both externally as 'tele-therapy' and internally as brachytherapy, or 'short-distance therapy', The number of cancer cases globally is expected to grow from 14.1 million in 2012 to 24 million by 2035 (Common Cancer Types - National Cancer Institute; Worldwide cancer data - World Cancer Research Fund). Improvements in cancer therapy, such as the dose enhancement by radiation and better computational methods for its estimation, the focus areas of research reported in this paper are thus of paramount importance to the science and technology of medicine. Of the 200 or more radioisotopes used in nuclear medicine (Radioisotopes in Medicine), common

\footnotetext{
* Corresponding author (umair.aziz@mail.au.edu.pk; (iD https://orcid.org/0000-0002-9554-0725)
} 
radioisotopes include technetium ${ }^{99 \mathrm{~m}} \mathrm{Tc}$, iodine ${ }^{125} \mathrm{I}$, palladium ${ }^{103} \mathrm{Pd}$, iridium ${ }^{192} \mathrm{Ir}$, cesium ${ }^{137} \mathrm{Cs}$, and cobalt ${ }^{60} \mathrm{Co}$.

Important radionuclides for brachytherapy include the conventional encapsulated (Perez-Calatayud et al., 2012) low dose rate (LDR) sources $(<200$ cGy per hour) Ir-192, and high dose rate (HDR) sources ( $>1200$ cGy per hour) (Pisansky et al., 2008; Mose et al., 2013; Mobit et al., 2016). Permanent brachytherapy sources (Lechtman et al., 2011) such as ${ }^{125} \mathrm{I}$ and ${ }^{103} \mathrm{Pd}$ with energy $<50 \mathrm{keV}$ are commonly used LDR sources, with typical implants of 50-80 metallic seeds encasing isotopes, used for the treatment of prostate cancer, uveal melanomas and brain tumours.

For calculations for dose distribution MC methods (Kalos \& Whitlock, 2008) and general-purpose codes, such as EGSnrc, GEANT (Allison et al., 2006), PENELOPE (Salvat et al., 2006) and MCNP (Team, 2008) have been extensively used (Jabbari, 2011; Seco, 2016) for simulating the transport of radiation from radiation seeds in the medium consisting of tissue, and material in the vicinity of the cancer. These codes have produced fast and accurate results which have been experimentally validated and benchmarked.

A further development in brachytherapy has been the possibility of injecting high-Z biocompatible elements such as gold and gadolinium in solution form through fenestrations of cancer cells to improve the effectiveness of therapy. The method thus requires elements of dimensions small enough to be delivered into tumour cells so that the effects of radiation are localized and restricted to the 'bad' cells while sparing the healthy 'good' cells. The determination of radiation dose, in addition to the previously mentioned factors, now extends to a determination of the choice of element, its size and shape, and injection procedure in solution form. The requirements of particle size necessitate the range down to nanoscale $\left(\sim 10^{-9} \mathrm{~m}\right)$ which compares with the diameter of an atom $\left(\sim 10^{-10} \mathrm{~m}\right)$, thus introducing nanotechnology to the field of brachytherapy (Jain et al., 2012; Chatterjee et al., 2013).

In brachytherapy, the use of gold nanoparticles (GNPs) is an active area of research to determine possible dose enhancement (Bahreyni Toossi et al., 2012; Brivio et al., 2017; Safigholi \& Song, 2018; Gray et al., 2019; Jangjoo et al., 2019) to make the therapy more effective. Nanoscale, at which the wavelength of incident electromagnetic radiation can become of the order of atomic dimensions casts doubts on the reliability of simulation results from Monte Carlo codes due to several artifacts.

The effects of such artifacts for electron transport have been investigated (Almansa et al., 2006; Koivunoro et al., 2012; Archambault \& Mainegra-Hing, 2015) with EGSnrc, GEANT and PENELOPE codes and in some cases 'large discrepancies' ( $>3 \%$ ) have been found between MCNP5 dose distributions and the 'reference codes' concluding that MCNP5 electron transport calculations are not accurate at all energies and in every medium by general clinical standards. Similarly, comparisons have been made (Yu et al., 2017) between EGSnrc, GEANT4, MCNP5 and PENELOPE for mono-energetic electron beams in a water-filled sphere of radius varying from $0.25-4.5 \mathrm{~cm}$ for beam energies of $0.5 \mathrm{MeV}, 1.0 \mathrm{MeV}$, and $5.0 \mathrm{MeV}$ and found to have differences below $10 \%$ by tuning parameters associated with multiple scattering algorithms at the expense of increased computation time. It can thus be anticipated that MCNP5 may differ due to its inadequate low-energy treatment of electron transport. Šidlová and Trojek (2010) have carried out electron transport comparisons of MCNPX, Penelope and EGSnrc, for electrons of 20-450 $\mathrm{keV}$ in water, lead and tungsten. These comparisons were focused on bremsstrahlung, energy deposition in matter, electron ranges and production of secondary electrons by gamma radiation. Thus, preliminary runs are carried out to compare dose estimates from MCNP5 with Penelope and Geant4 (Almansa et al., 2006).

Thus, benchmarking and validating $\mathrm{MC}$ results have become particularly relevant not only for radiation dose but also for the resulting biological absorbed dose, which is the ultimate quantity of interest varying from organ to organ. With the objective of determining radiation dose distribution and the dose enhancement factor (DEF) by the use of gold, a high- $Z$ biocompatible element, this work carries out MC simulation, using MCNP5, for simulating coupled photon-electron radiation transport from $\mathrm{x}$-rays emanating from a radiation source implanted in tumour, modelled by a spherical water phantom, to estimate the energy deposited and the subsequent biological dose. Good comparisons are presented for point mono-energetic source in water (Luxton \& Jozsef, 1999; Almansa et al., 2006). While water phantoms are used for representing configurations similar to soft tissue, full anthropomorphic phantoms are also used to carry out detailed simulation for the dose (Neves et al., 2014)

For MC simulations in brachytherapy (Lechtman et al., 2013; Mesbahi et al., 2013; Asadi et al., 2015) it has been shown that photon source energy and 
concentration are important factors that determine DEF while the size of GNPs is not so important (above the K-edge energy). Thus, in this work, a homogenous model with considerable savings on the computational effort, as well as a sensitivity capability is used to extract crucial information on the DEF from independent parameters.

The MCNPX code has been used (Mesbahi et al., 2013) for simulating mono-energetic photon beams (50-120 keV), ${ }^{60} \mathrm{Co}$ beam, and 6 \& $18 \mathrm{MV}$ photon beams, while GEANT4 simulations have been carried out (Banoqitah \& Djouider, 2016) for estimating the DEF from high energy photons from ${ }^{192} \operatorname{Ir}(0.380 \mathrm{MeV})$, ${ }^{137} \mathrm{Cs}(0.662 \mathrm{MeV})$ and ${ }^{60} \mathrm{Co}(1.2 \mathrm{MeV})$ for gadolinium $(Z=64)$ nanoparticles in a heterogeneous model with concentrations ranging from $10-70 \mathrm{mg} / \mathrm{g}$ brain tumour.

In this work, the source energy of interest is 15 $\mathrm{keV}-1 \mathrm{MeV}$ typical of brachytherapy sources $(15 \mathrm{keV}$, $20 \mathrm{keV}, 30 \mathrm{keV}, 40 \mathrm{keV}, 80 \mathrm{keV}, 90 \mathrm{keV}, 150 \mathrm{keV}, 300$ $\mathrm{keV}, 1 \mathrm{MeV}$ ), while the gold particles in solution form with water were assumed to comprise a homogenous mixture with concentrations in the range 5-25 mg Au/g $\mathrm{H}_{2} \mathrm{O}$. While MCNP has the capability of modelling very detailed heterogeneous configurations, this paper considers a homogenous model solely to demonstrate (i) results from benchmarked problems and (ii) for obtaining MC sensitivity estimates for a 'bulk' material to demonstrate validity of MC perturbation and quantifying increased computational efficiency.

For comparison, dose estimates from MCNP5 were compared with published results from PENELOPE and GEANT. The second objective of this work was to estimate the effect of small changes in solution concentration on the resulting radiation dose from a single MC simulation rather than estimating changes from MC re-runs where the effect is vulnerable to be masked in the uncertainty of the estimate itself. Such MC perturbation analysis has applications in brachytherapy for estimating dose perturbations when implants are present in the vicinity of an organ receiving radiation from an implanted source (Yu et al., 2017). However, it is yet to be used in simulation for brachytherapy studies and can provide great computational efficiency leading to optimal designs based on 'best' experimental parameters such as radiation energy, concentration of gold in solution and material placement for maximizing an objective function of interest.

There is still no consensus on the optimal size, shape and distribution of GNPs. Experimental and pre-clinical evidence for mouse tumours showing a 1-year survival rate of $86 \%$ following a dose of $26 \mathrm{~Gy}$, with $1.9 \mathrm{~nm}$ intravenously administered GNPs vs 20\% for tumours not laden with GNPs (Jain et al., 2012), indicates that nanotechnology offers promising improvements in brachytherapy. This work is aimed to further improve the understanding of determining possible improvements in delivered dose by applying Monte Carlo simulation, and the MC perturbation capability (Rief, 1984; Koreshi \& Lewins, 1990), to lead to optimal experimental configurations.

\section{METHODOLOGY}

\section{Monte Carlo simulation}

The Monte Carlo code MCNP5 is used to carry out a coupled photon-electron simulation of radiation transport to estimate the dose distribution, from a point isotropic photon source typical of brachytherapy sources $(30 \mathrm{keV}$ for ${ }^{125} \mathrm{I}-1.25 \mathrm{MeV}$ for ${ }^{60} \mathrm{Co}$ ) located at the origin of a sphere, in concentric shells of water of thickness $0.5 \mathrm{~mm}$ up to a radius of $15 \mathrm{~cm}$. A solution of gold and water is then considered in the shells located $1-2 \mathrm{~cm}$ from the center and the F6 (energy deposition) and *F8 (pulse height) tallies are used for phantom dosimetry. Photon and electron data for air, water and gold are based on ENDF/B-VI (Release 8). In the MCNP 'detailed physics' simulation, coherent (Thomson) scattering is included, and fluorescent photons produced from photoelectric absorption are included and electrons produced from photon collisions are transported in a 'condensed history' method that accumulates the effects of many individual collisions into single steps sampled probabilistically.

The F6 tally given as equation (1)

$D=\frac{\rho_{a}}{m} \int d E \int d t \int d V \int d \Omega \sigma_{t}(E) H(E) \phi(\bar{r}, \widehat{\Omega}, E, t) \mathrm{MeV} \mathrm{g}^{-1}$

where $D$ is dose, $m$ is mass, $\rho_{a}$ is atomic density, $t$ is time, $E$ is energy, $V$ is volume, $\Omega$ is solid angle, $\phi$ is scalar flux scored over volume (track length/volume) $\mathrm{cm}^{-2}, \sigma_{t}$ is microscopic total cross-section and $H$ is heating number (MeV/collision),

from $\mathrm{MCNP}$ is converted from $\mathrm{MeV} / \mathrm{g}$ to yield the dose in $\mathrm{nGy} \mathrm{cm}^{2} \mathrm{~Bq}^{-1} \mathrm{hr}^{-1}$ to compare with Almansa et al. (2006). The results plotted are for $\dot{D}=\kappa r^{2} D \mathrm{nGy} \mathrm{cm}{ }^{2} \mathrm{~Bq}^{-1} \mathrm{hr}^{-1}$ where $\kappa=576 \mathrm{nGy} \mathrm{cm}^{2} \mathrm{~Bq}^{-1} \mathrm{hr}^{-1} /\left(\frac{\mathrm{MeV}}{\mathrm{g}}\right)$.

\section{Monte Carlo perturbation sampling}

Perturbation algorithms in MC simulation (Rief, 1984) extended the capability of MC methods to sensitivity studies and optimization (Koreshi \& Lewins, 1990). The 
change in a response function such as dose $D$, due to a variation in an independent parameter, such as material density $\rho$, expressed as a Taylor series in equation (2)

$D(\rho)=D\left(\rho_{o}\right)+D^{\prime}\left(\rho_{o}\right) \delta \rho+\frac{1}{2 !} D^{\prime \prime}(\delta \rho)^{2}+\cdots$

can be used with first- and second-order derivatives $D^{\prime}$ and $D^{\prime \prime}$ from a single run. Thus, when the change in a parameter is small enough to be of the order of the statistical uncertainty of a MC estimate, then the difference from two independent runs may mask the actual difference. In order to circumvent such inaccuracy, the derivatives are sampled in a single simulation. The energy deposition for a 'perturbed' design $\left(D^{\text {new }}\right)$ can thus be estimated from a Taylor series in terms of a 'reference' design ( $\left.D^{\text {ref }}\right)$.

\section{Material composition}

The material used in this work is water with gold solution which has density $\rho=1.04 \mathrm{~g} \mathrm{~cm}^{-3}$ very close to that of soft tissue $\rho=1.04 \mathrm{~g} \mathrm{~cm}^{-3}$ [with a four-component simplified composition hydrogen $(\mathrm{H})$, carbon $(\mathrm{C})$, nitrogen $(\mathrm{N})$ and oxygen $(\mathrm{O})$ with weight percentages: $10.454,22.663,2.49,63.525 \%$, respectively] and the photon cross-sections for both (water and soft tissue) are almost indistinguishable so that conclusions drawn for energy deposition would hold for both materials to a reasonably acceptable order.

\section{RESULTS AND DISCUSSION}

\section{Benchmarking of radiation dose and dose distribution}

To compare MCNP5 results for energy deposition from a mono-energetic point isotropic source at the centre of a sphere of radius $15 \mathrm{~cm}$, the $\mathrm{F} 6$ tally in $0.5 \mathrm{~mm}$ thick concentric shells were used with 1 million histories for each source energy level. From the results shown in Figure 1, it is seen that there is good agreement with the results given in the literature (Almansa et al., 2006). When normalized to a reference dose from a $1 \mathrm{MeV}$ source, it is seen that the lowest energy, $15 \mathrm{keV}$, has an energy deposition of about $65 \%$ of the reference which falls off rapidly to less than $10 \%$ in $1.5 \mathrm{~cm}$ of water. With a further increase in energy, the relative intensities drop but the deposition sustains to greater depths, which is less important in brachytherapy than to give a localized deposition from a low-energy source.

MCNP simulations were carried out on an Intel(R) Core (TM) i7-2620M CPU @ 2.70GHz with an installed memory of 8.00 GB (3.24 GB usable) and 32-bit Operating system with Windows 7 Professional.

The DEF shown in Figure 2 gives the extent and magnitude as a function of source energy and concentration confirming the trend of direct variation with concentration and inverse variation with source

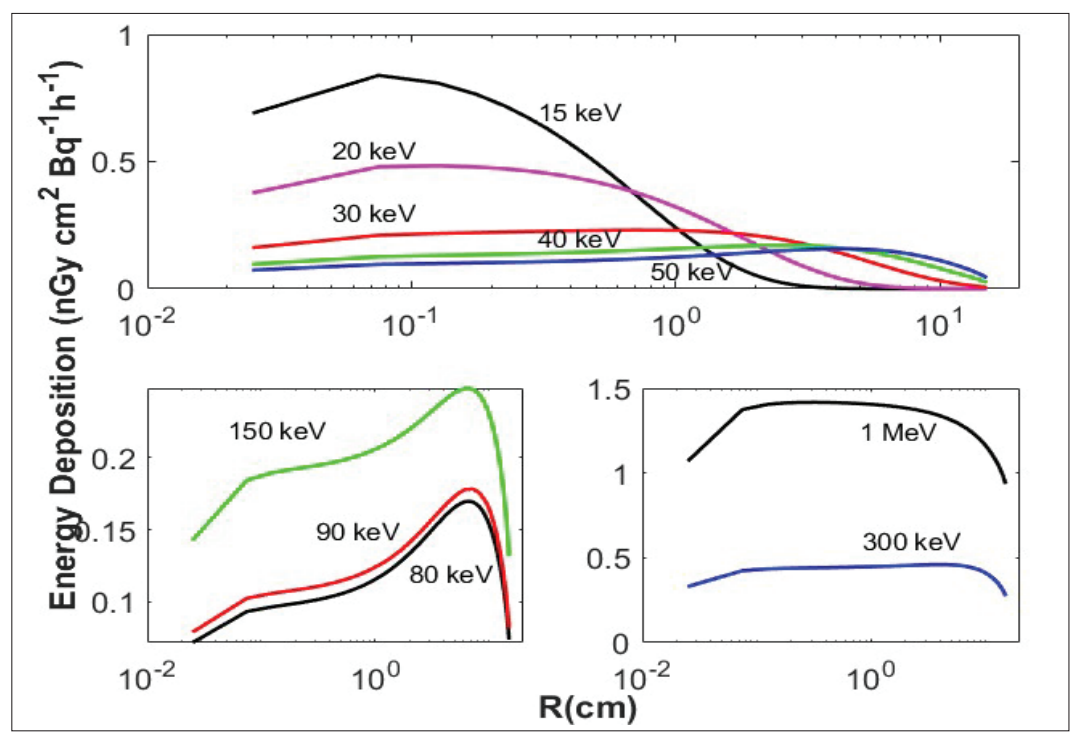

Figure 1: Energy deposition for mono-energetic photons of energy E: $15 \mathrm{keV}^{-1} \mathrm{MeV}$ 


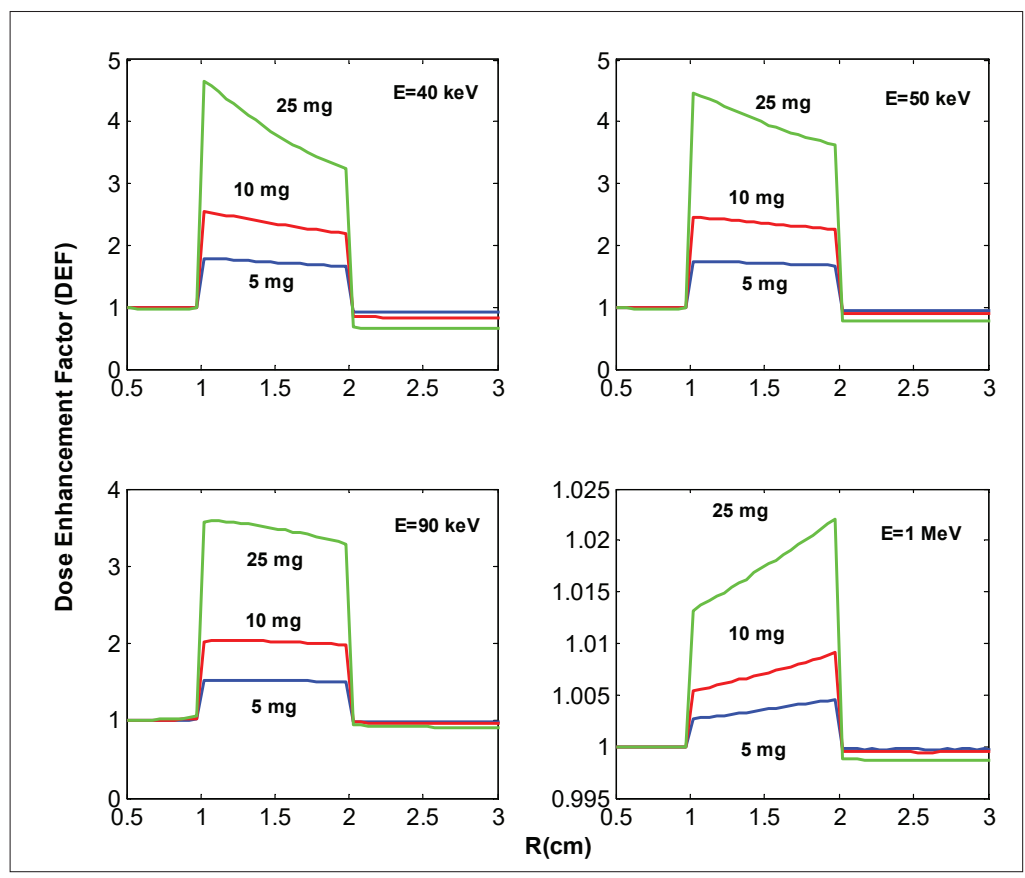

Figure 2: Dose enhancement factor (DEF) vs. distance for $\mathrm{E}=40 \mathrm{keV}, 50 \mathrm{keV}$, $90 \mathrm{keV}, 1 \mathrm{MeV}$ for varying concentrations 5, 10, $25 \mathrm{mg} \mathrm{Au} / \mathrm{g} \mathrm{H}_{2} \mathrm{O}$

energy. The rate of 'fall' or 'rise' of DEF in the solution region in indicative of the slightly longer spatial effect of the high-energy photons as compared to low-energy photons.

For ophthalmic brachytherapy with ${ }^{125}$ I (mean energy $35.49 \mathrm{keV}$ ), a full three-dimensional heterogeneous model (Asadi et al., 2015) simulating $10^{7}-10^{9}$ histories, for $50 \mathrm{~nm}$ GNPs, estimate the DEF for 7, 10, 18 and 30 $\mathrm{mg} / \mathrm{g}$ as $1.9,2.2,3.2$ and 4.6 for the tumour phantom compared with 1.9, 2.3, 3.3 and 4.8 in the water phantom, respectively.

As given in Table 1, this work, for a water phantom with $10^{6}$ simulations with a maximum time $\sim 35$ minutes in a homogenous mixture model for the solution gives, for $40 \mathrm{keV}$ and concentrations of $5,10,25 \mathrm{mg} / \mathrm{g}$ maximum DEFs (Figure 3) of 1.8, 2.5. 4.6. DEF has been found to lie in the range 1.4-3.7 with the highest DEF for 90 $\mathrm{keV}$ (Mesbahi et al., 2013); the effect of GNP size was not considerable (Lechtman et al., 2011; Mesbahi et al., 2013) while concentration and energy were important. These simulations (Lechtman et al., 2011; Mesbahi et al., 2013) carried out for 7 and $18 \mathrm{mg} \mathrm{Au} / \mathrm{g} \mathrm{H}_{2} \mathrm{O}$ for 30, 50, $100 \mathrm{~nm}$ GNPs and energies between $50-18 \mathrm{MeV}$, report highest DEF of 3.5 at $90 \mathrm{keV}$ followed by 3.0 at $50 \mathrm{keV}$ both for $30 \mathrm{~nm}$ GNPs and $18 \mathrm{mg} / \mathrm{g}$ concentration.
Table 1: Max DEF for $1 \mathrm{M}$ simulations vs $\mathrm{E}$ and concentration; CPU time (mins) shown in brackets

\begin{tabular}{ccccc}
\hline No. & $\mathrm{E}(\mathrm{keV})$ & $\alpha=5$ & $\alpha=10$ & $\alpha=25$ \\
\hline \multirow{2}{*}{1} & \multirow{2}{*}{40} & 1.7854 & 2.5398 & 4.6537 \\
& & $(33.17)$ & $(35.48)$ & $(17.86)$ \\
2 & \multirow{2}{*}{50} & 1.7394 & 2.4521 & 4.4529 \\
& & $(40.53)$ & $(42.47)$ & $(21.56)$ \\
3 & \multirow{2}{*}{90} & 1.5204 & 2.0398 & 3.5861 \\
& & $(59.56)$ & $(58.19)$ & $(31.02)$ \\
4 & \multirow{2}{*}{1000} & 1.0046 & 1.0091 & 1.0221 \\
& & $(98.71)$ & $(101.78)$ & $(71.46)$ \\
\hline
\end{tabular}

For high energy sources, with $10^{8}$ source particles simulated, it is reported (Banoqitah \& Djouider, 2016) that a maximum DEF is 1.45 within the tumour when implanted with $70 \mathrm{mg} / \mathrm{g}$ Gd for the lowest energy $\left({ }^{192} \mathrm{Ir}\right)$, and that the DEF varies directly with concentration and inversely with source energy e.g. for a concentration of $30 \mathrm{mg} / \mathrm{g}$ it decreases from 1.24 to 1.09 when photon energy increases from $0.380 \mathrm{MeV}\left({ }^{192} \mathrm{Ir}\right)$ to $1.20 \mathrm{MeV}$ $\left({ }^{60} \mathrm{Co}\right)$. The results for this work show similar trends at high energy $(1 \mathrm{MeV})$, i.e., a maximum DEF increasing very slowly from 1.0046 to 1.0221 as concentration increases from $5 \mathrm{mg} / \mathrm{g}$ to $25 \mathrm{mg} / \mathrm{g}$. 


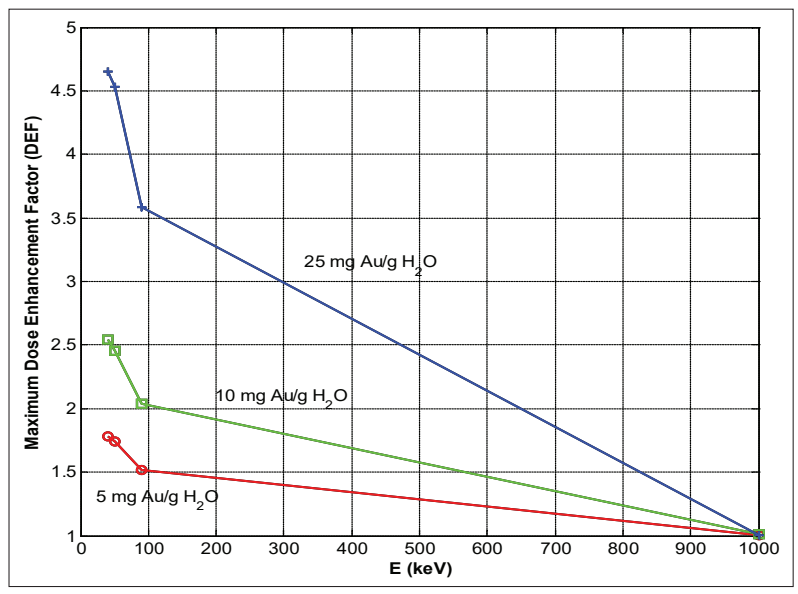

Figure 3: Maximum dose enhancement factor vs. energy for varying concentrations 5, 10, $25 \mathrm{mg} \mathrm{Au} / \mathrm{g} \mathrm{H}_{2} \mathrm{O}$

\section{MC perturbation estimates}

The simulations carried out for the results presented in previous sections were computationally intensive, which usually requires in excess of one hour for high energy $(1 \mathrm{MeV})$. Thus, MC would be inefficient for carrying out sensitivity studies where the effect of perturbations, such as the solution concentration of gold in water, on the DEF would be required. If a change is small then the statistical uncertainty in the results from two independent simulations, from which the change in the outcome is being estimated, could mask the effect of the change and hence produce unreliable results. Thus, perturbation MC was examined to obtain first- and second-order derivative changes, shown in Figure 4, in concentration of the gold solution in the range 5-25 mg Au/g $\mathrm{H}_{2} \mathrm{O}$. For this, a single run at a reference design of $5 \mathrm{mg} \mathrm{Au} / \mathrm{g} \mathrm{H}_{2} \mathrm{O}$ was carried out and derivatives were obtained for the energy deposition to predict results for $15 \mathrm{mg} \mathrm{Au} / \mathrm{g} \mathrm{H}_{2} \mathrm{O}$ in 35.24 minutes compared with 33.17 minutes as given in Table 1.

The $1^{\text {st }}$ and $2^{\text {nd }}$ order perturbation results, $15 \mathrm{mg}$ (1PT) and $15 \mathrm{mg}$ (2PT) respectively, for estimating the energy deposition at $15 \mathrm{mg} / \mathrm{g}$ are almost identical due to the small magnitude of the perturbation rendering the $2^{\text {nd }}$ derivative an almost insignificant contribution. A better idea of the differences from re-run estimates $(\Delta D)_{r}$ compared with perturbation theory estimates $(\Delta D)_{p}$ is depicted in Figure 5, where the ratio $\gamma$, given by equation (3), remains $\sim 1$ in the gold solution region $(1-2 \mathrm{~cm})$ but at the boundary where there is a sharp jump, the perturbation estimates are also unreliable, since the magnitude of the perturbation exceeds the validity of $2^{\text {nd }}$ order Taylor series.

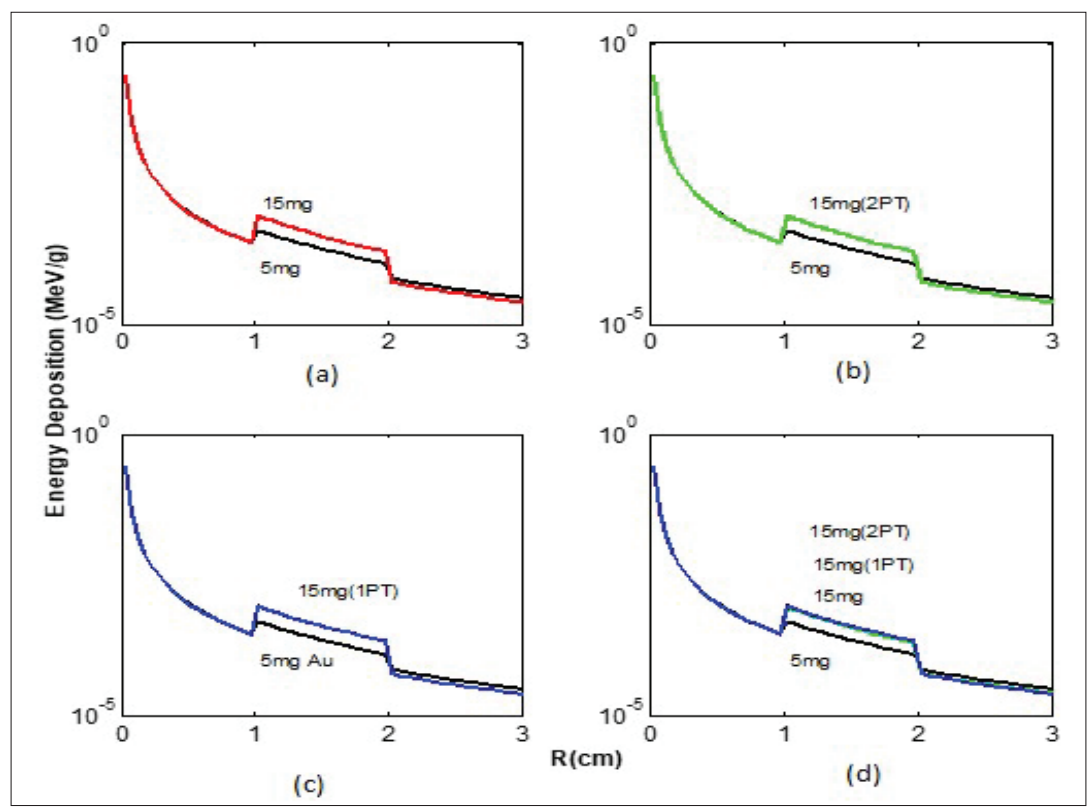

Figure 4: Energy deposition (MeV/g) vs. distance for photon energy $40 \mathrm{keV}$ : (a) two independent MC simulations; (b) $5 \mathrm{mg}$ simulation compared with $2^{\text {nd }}$ order perturbation theory estimate for $15 \mathrm{mg}$ perturbed case; (c) $5 \mathrm{mg}$ simulation compared with $1^{\text {st }}$ order perturbation theory estimate for $15 \mathrm{mg}$ perturbed case; (d) all four cases 


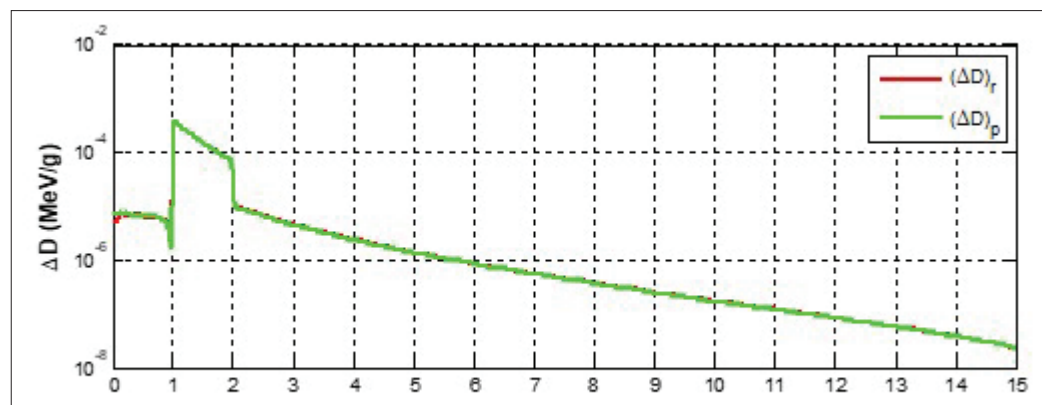

(a)

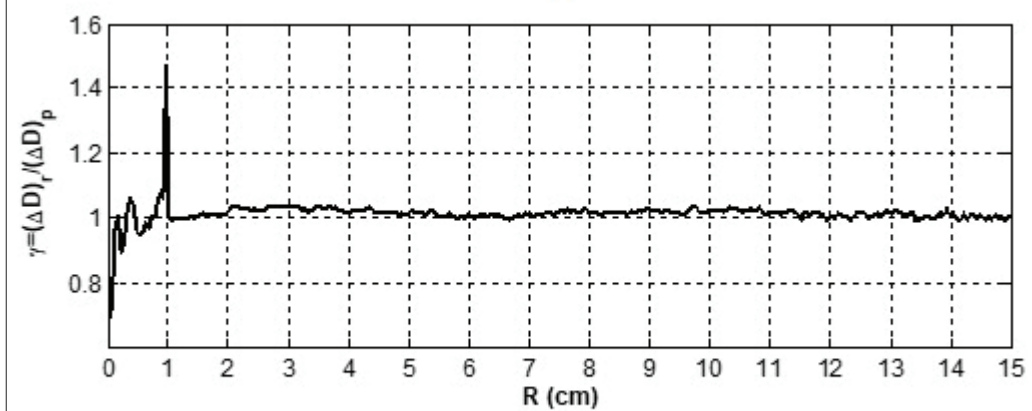

(b)

Figure 5: Energy deposition (MeV/g) vs. distance for photon energy $40 \mathrm{keV}$ : (a) differences from independent re-runs $(\Delta \mathrm{D})_{\mathrm{r}}$ and differences based on perturbation estimate $(\Delta \mathrm{D})_{\mathrm{p}} ;$ (b) ratio of differences $\gamma$

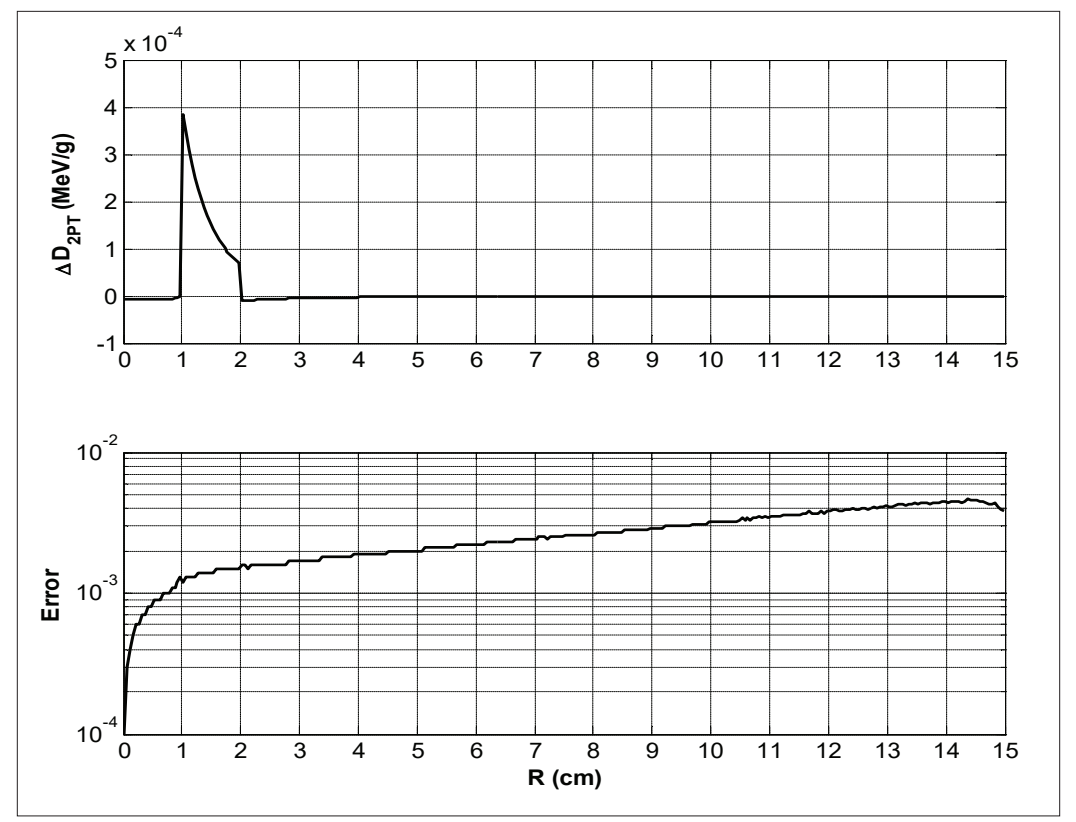

Figure 6: Differences in energy deposition $(\mathrm{MeV} / \mathrm{g})$ vs. distance for photon energy $40 \mathrm{keV}$ : (a) differences based on $2^{\text {nd }}$ order perturbation estimate $(\Delta D)_{p}$; (b) standard errors in estimates of $(\Delta \mathrm{D})_{\mathrm{p}}$ 
$\gamma \equiv \frac{D^{\text {new }}-D^{\text {ref }}}{D_{p}^{\text {new }}-D^{\text {ref }}}$

A further test of the reliability of the results is given in Figure 6 which shows the magnitude of the second-order perturbation estimate and the errors $R$ for which the $68 \%$ confidence limit of a result is where is the estimate of the change in estimated dose from $2^{\text {nd }}$ order $\mathrm{MC}$ perturbation sampling, increasing from $10^{-4}$ to $\sim 5 \times 10^{-3}$ at the outer edge of the sphere.

\section{CONCLUSIONS}

In this work, the dose enhancement by injecting gold in solutions was estimated as a function of source energy and solution concentration. The important photon interaction for materials with high atomic number $(Z)$ is the photoelectric effect for which the cross-section varies as $\mu_{P E} \sim \frac{\rho Z^{3}}{E^{3}}$ (where $\mu_{P E}$ is photoelectric attenuation coefficient) so that low energy and high- $Z$ are desirable for dose enhancement which is localized to the tumour due to the short range of photoelectrons and Auger electrons in the surrounding medium which for electrons of energy $0.1 \mathrm{MeV}$ is $\sim 100$ microns in water and $\sim 15$ microns in gold so that the effect of gold will require thin layers (of the order of to utilize the energy of photoelectrons in water. At this energy, the photoabsorptions were estimated to be $\sim 13.5 \%$ of all interactions.

Computational efficiency in MC simulation for estimating small changes in parameters has been demonstrated using a perturbation feature of $\mathrm{MC}$ simulation, thereby greatly reducing the computational effort to perform simulations in brachytherapy, extending its applicability to full-scale heterogeneity sensitivity studies pertinent to the modelling of nanoparticles in implanted solutions.

Important conclusions from this research were: (i) MCNP dose estimates were found to be in acceptable agreement with PENELOPE and GEANT in spite of artifacts such as cut-offs in electron transport (Archambault \& Mainegra-Hing, 2015), (ii) dose increases with energy of a source, (iii) dose enhancement, for a given concentration, decreases with source energy, and (iv) MC perturbation theory is valid and useful, due to enhanced computational efficiency, for the practically important range of material density in solutions of gold and water, assumed homogenously mixed.

\section{Conflict of interest}

Authors declare that they have no conflict of interest.

\section{REFERENCES}

Allison J. et al. (2006). Geant4 developments and applications. IEEE Transactions on Nuclear Science 53(1): 270-278. DOI: https://doi.org/10.1109/TNS.2006.869826

Almansa J.F., Guerrero R., Al-Dweri F.M.O., Anguiano M. \& Lallena A.M. (2006). Dose distribution in water for monoenergetic photon point sources in the energy range of interest in brachytherapy: Monte Carlo simulations with PENELOPE and GEANT4. Nuclear Instruments and Methods in Physics Research B 254: 219-230.

Archambault J.P. \& Mainegra-Hing E. (2015). Comparison between EGSnrc, Geant4, MCNP5 and Penelope for mono-energetic electron beams. Physics in Medicine and Biology 60(13): 4951-4962.

DOI: https://doi.org/10.1088/0031-9155/60/13/4951

Asadi S., Vaez-zadeh M., Masoudi S.F., Rahmani F., Knaup C. \& Meigooni A.S. (2015). Gold nanoparticle-based brachytherapy enhancement in choroidal melanoma using a full Monte Carlo model of the human eye. Journal of Applied Clinical Medical Physics 16(5): 344-357.

DOI: https://doi.org/10.1120/jacmp.v16i5.5568

Bahreyni Toossi M.T., Ghorbani M., Mehrpouyan M., Akbari F., Sobhkhiz Sabet L. \& Soleimani Meigooni A. (2012). A Monte Carlo study on tissue dose enhancement in brachytherapy: a comparison between gadolinium and gold nanoparticles. Australasian Physical and Engineering Sciences in Medicine 35(2): 177-185.

DOI: https://doi.org/10.1007/s13246-012-0143-3

Banoqitah E. \& Djouider F. (2016). Dose distribution and dose enhancement by using gadolinium nanoparticles implant in brain tumor in stereotactic brachytherapy. Radiation Physics and Chemistry 127: 68-71.

DOI: https://doi.org/10.1016/j.radphyschem.2016.06.002

Brivio D., Nguyen P.L., Sajo E., Ngwa W. \& Zygmanski P. (2017). A Monte Carlo study of I-125 prostate brachytherapy with gold nanoparticles: dose enhancement with simultaneous rectal dose sparing via radiation shielding. Physics in Medicine and Biology 62(5): 19351948.

DOI: https://doi.org/10.1088/1361-6560/aa5bc7

Chatterjee D.K., Wolfe T., Lee J., Brown A.P., Singh P.K., Bhattarai S.R., Diagaradjane P. \& Krishnan S. (2013). Convergence of nanotechnology with radiation therapyinsights and implications for clinical translation. Translational cancer research 2(4): 256-268.

DOI: https://doi.org/10.3978/j.issn.2218-676X.2013.08.10

Common Cancer Types. National Cancer Institute. Available at https://www.cancer.gov/types/common-cancers

Gray T., Mayer K., Kirby N. \& Team T.G. (2019). Computational 
assessment of radiation dose enhancement and secondary electron production for variable sizes and concentrations of gold nanospheres in a tumor using MCNP6.2. APS March Meeting. Abstract id. P23.006. Available at https:// ui.adsabs.harvard.edu/abs/2019APS..MARP23006G

Jabbari K. (2011). Review of fast Monte Carlo codes for dose calculation in radiation therapy treatment planning. Journal of Medical Signals and Sensors 1(1): 73-86.

Jain S., Hirst D.G. \& O'Sullivan J.M. (2012). Gold nanoparticles as novel agents for cancer therapy. The British Journal of Radiology 85(1010): 101-113.

DOI: https://doi.org/10.1259/bjr/59448833

Jangjoo A.G., Ghiasi H. \& Mesbahi A. (2019). A Monte Carlo study on the radio-sensitization effect of gold nanoparticles in brachytherapy of prostate by $103 \mathrm{Pd}$ seeds. Polish Journal of Medical Physics and Engineering 25(2): 87-92. DOI: https://doi.org/10.2478/pjmpe-2019-0012

Kalos M.H. \& Whitlock P.A. (2008). Monte Carlo Methods. Wiley-Blackwell, USA.

Koivunoro H., Siiskonen T., Kotiluoto P., Auterinen I., Hippeläinen E. \& Savolainen S. (2012). Accuracy of the electron transport in menp5 and its suitability for ionization chamber response simulations: A comparison with the EGSNRC and PENELOPE codes. Medical Physics 39(3): 1335-1344.

DOI: https://doi.org/10.1118/1.3685446

Koreshi Z.U. \& Lewins J.D. (1990). Two-group Monte Carlo perturbation theory and applications in fixed-source problems. Progress in Nuclear Energy 24(1-3): 27-38. DOI: https://doi.org/10.1016/0149-1970(90)90020-6

Lechtman E., Chattopadhyay N., Cai Z., Mashouf S., Reilly R. \& Pignol J.P. (2011). Implications on clinical scenario of gold nanoparticle radiosensitization in regard to photon energy, nanoparticle size, concentration and location. Physics in Medicine and Biology 56(15): 4631-4647. DOI: https://doi.org/10.1088/0031-9155/56/15/001

Lechtman E., Mashouf S., Chattopadhyay N., Keller B.M., Lai P., Cai Z., Reilly R.M. \& Pignol J.P. (2013). A Monte Carlo-based model of gold nanoparticle radio sensitization accounting for increased radiobiological effectiveness. Physics in Medicine and Biology 58(10) 3075-3087. DOI: https://doi.org/10.1088/0031-9155/58/10/3075

Luxton G. \& Jozsef G. (1999). Radial dose distribution, dose to water and dose rate constant for monoenergetic photon point sources from $10 \mathrm{keV}$ to $2 \mathrm{MeV}$ : EGS4 Monte Carlo model calculation. Medical Physics 26(12): 2531-2538. DOI: https://doi.org/10.1118/1.598790

Mesbahi A., Jamali F. \& Garehaghaji N. (2013). Effect of photon beam energy, gold nanoparticle size and concentration on the dose enhancement in radiation therapy. BioImpacts:BI 3(1): 29-35.

DOI: https://doi.org/10.5681/bi.2013.002

Mobit P.N., Nguyen A., Packianathan S., He R. \& Yang C.C. (2016). Dosimetric comparison of brachytherapy sources for high dose-rate treatment of endometrial cancer: 192Ir, $60 \mathrm{Co}$ and an electronic brachytherapy source. British Journal of Radiology 89(1059).

DOI: https://doi.org/10.1259/bjr.20150449
Neves L.P., Santos W.S., Gorski R., Perini A.P., Maia A.F., Caldas L.V.E. \& Orengo G. (2014). Dosimetric study of a brachytherapy treatment of esophagus with Brazilian 192Ir sources using an anthropomorphic phantom. Radiation Physics and Chemistry 104: 240-243.

DOI: https://doi.org/10.1016/j.radphyschem.2013.11.029

Perez-Calatayud J., Ballester F., Das R.K., Dewerd L.A., Ibbott G.S., Meigooni A.S., Ouhib Z., Rivard M.J., Sloboda R.S. \& Williamson J.F. (2012). Dose calculation for photonemitting brachytherapy sources with average energy higher than $50 \mathrm{keV}$ : Report of the AAPM and ESTRO. Medical Physics 39(5): 2904-2929.

DOI: https://doi.org/10.1118/1.3703892

Pisansky T.M., Gold D.G., Furutani K.M., Macdonald O.K., McLaren R.H. Mynderse L.A, Wilson T.M., Hebl J.R. \& Choo R. (2008). High-dose-rate brachytherapy for localized prostate cancer. Mayo Clinic Proceedings 83(12): 1364.

Radioisotopes in Medicine. Nuclear Medicine - World Nuclear Association. Available at http://www.world-nuclear.org/ information-library/non-power-nuclear-applications/ radioisotopes-research/radioisotopes-in-medicine.aspx

Rief H. (1984). Generalized Monte Carlo perturbation algorithms for correlated sampling and a second-order Taylor series approach. Annals of Nuclear Energy 11(9):455-476.

DOI: https://doi.org/10.1016/0306-4549(84)90064-1

Safigholi H. \& Song W.Y. (2018). Macroscopic and Microscopic dose enhancement factor (def) for tumor diffusion with gold nanoparticles (gnps) using Monte Carlo simulations: $103 \mathrm{Pd}, 125 \mathrm{I}$, and electronic brachytherapy (ebt) sources. Brachytherapy 17(4): S32-S33.

DOI: https://doi.org/10.1016/j.brachy.2018.04.044

Salvat F., Fernández-Varea J.M. \& Sempau J. (2006). PENELOPE2008: a code system for monte carlo simulation of electron and photon transport. Available at http://www. oecd-nea.org/lists/penelope.html

Saw C. B. (2013). Brachytherapy: High Dose Rate (HDR) Implants. In: Encyclopedia of Radiation Oncology (L. W. Brady \& T. E. Yaeger (eds.)). Springer, Berlin, Heidelberg. DOI: https://doi.org/10.1007/978-3-540-85516-3_143

Seco J. (2016). Monte Carlo Techniques in Radiation Therapy: CRC Press, USA.

Š́́dlová V. \& Trojek T. (2010). Testing Monte Carlo computer codes for simulations of electron transport in matter. Applied Radiation and Isotopes 68(4-5): 961-964. DOI: https://doi.org/10.1016/j.apradiso.2009.12.019

Team X.-M.C. (2008). MCNP-A General MC N-Particle Transport Code, Version 5. 836.

Worldwide Cancer Data. World Cancer Research Fund. American Institute for Cancer Research. Available at https://www.wcrf.org/dietandcancer/cancer-trends/ worldwide-cancer-data

Yu P.C., Nien H.H., Tung C.J., Lee H.Y., Lee C.C., Wu C.J. \& Chao T.C. (2017). CT-based MCNPX dose calculations for gynecology brachytherapy employing a Henschke applicator. Radiation Physics and Chemistry 140: 392-397. DOI: https://doi.org/10.1016/j.radphyschem.2017.03.044 